\title{
Arbeitsplatzbezogene Belastungen und Ressourcen von ausländischen 24-Stunden-Betreuungskräften in deutschen Privathaushalten - eine qualitative Studie
} Psychosocial Stressors and Resources of Live-in Caregivers in
German Households - a Qualitative Study

(c) (1) $\odot \odot$

\author{
Autoren \\ Benjamin Schilgen ${ }^{1}$, Katja Savcenko², Albert Nienhaus ${ }^{3,4}$, Mike Mösko
}

Institute

1 Institut und Poliklinik für Medizinische Psychologie, Universitätsklinikum Hamburg-Eppendorf, Hamburg

2 Institut für Psychologie, Universität Koblenz-Landau, Koblenz

3 Competenzzentrum Epidemiologie und Versorgungsforschung bei Pflegeberufen, Universitätsklinikum Hamburg-Eppendorf, Hamburg

4 Berufsgenossenschaft für Gesundheitsdienst und Wohlfahrtspflege, Abteilung Arbeitsmedizin, Gefahrstoffe und Gesundheitswissenschaften, Hamburg

\section{Schlüsselwörter}

24-Stunden-Betreuungskräfte, Privathaushalte, arbeitsplatzbezogene Gesundheit, Belastungen, Ressourcen, irreguläre Beschäftigung

Key words

Live-in caregivers, irregular employment, Workload, Home nursing, Occupational health, Qualitative research

\section{Bibliografie}

DOI https://doi.org/10.1055/a-1005-7024

Online-Publikation: 7.11.2019

Gesundheitswesen 2020; 82: 196-201

(c) Georg Thieme Verlag KG Stuttgart · New York ISSN 0941-3790

Korrespondenzadresse

Benjamin Schilgen

Institut und Poliklinik für Medizinische Psychologie

Universitätsklinikum Hamburg-Eppendorf

Martinistraße 52

20246 Hamburg

b.schilgen.ext@uke.de

\section{ZUSAMMENFASSUNC}

Ziel der Studie 24-Stunden-Betreuungskräfte, die in deutschen Privathaushalten haushalts- und personennahe Dienstleistungen für ältere pflegebedürftige Menschen erbringen, stammen meist aus dem Ausland. Diese Arbeitsverhältnisse liegen in der Regel außerhalb tariflich oder arbeitsrechtlich verbindlicher Vereinbarungen. Betreuungskräfte erfahren soziale Isolation sowie eine Vermischung von Arbeit und Privatsphäre. Die Studienlage ist aufgrund der schwierigen Erreichbarkeit der Zielgruppe sehr lückenhaft. Die vorliegende Studie ist eine qualitative explorative Analyse der Arbeits- und Gesundheitssituation von 24-Stunden-Betreuungskräften mit dem Fokus auf arbeitsplatzbezogene Belastungen und Ressourcen.

Methodik Die Rekrutierung erfolgte über Vermittlungsagenturen, Onlineportale, soziale Netzwerke und Vereine. Es wurden 9 semistrukturierte Interviews mit ausländischen 24-Stunden-Betreuungskräften in Privathaushalten geführt. Die Auswertung orientierte sich an der zusammenfassenden Inhaltsanalyse nach Mayring mit einer induktiven Kategorienbildung.

Ergebnisse und Schlussfolgerung Ständige Verfügbarkeit, Verständigungsschwierigkeiten und interpersonelle Konflikte sind alltägliche Belastungen für ausländische 24-Stunden-Betreuungskräfte. Diese Belastungen gehen mit Beeinträchtigungen der psychischen und physischen Gesundheit in Form von Erschöpfung, Schlaflosigkeit, erhöhter Reizbarkeit und Depressionssymptomen einher. Die Probandinnen erfahren aber auch Unterstützung durch Dritte und können sich emotional und räumlich von der direkten Betreuungssituation distanzieren. Das Erleben von Dankbarkeit, die Erfahrung akzeptiert zu werden aber auch eine finanzielle Absicherung werden als weitere relevante Ressourcen genannt. Die Sichtbarmachung der Zielgruppe ist forschungsbezogen durch einen ressourcenintensiven Rekrutierungsaufwand und politisch durch die Einführung einer gemeinsamen Berufsdefinition zu gewährleisten.

\section{ABSTRACT}

Aim of the study Live-in caregivers who provide householdand person-related services for the elderly in need of care in German private households mostly come from abroad. These labour conditions generally do not meet any tariff or labour law-related binding regulations. Live-in caregivers are additionally exposed to social isolation and the confusion of work and privacy. The study is full of gaps because the target group cannot 
be reached easily. The aim of the present study was to qualitatively explore the work and health situation of live-in caregivers with a focus on workplace-related stressors and resources.

Methodology The recruitment process was carried out via placement agencies, online portals, social network platforms as well as care associations. Nine semi-structured interviews were conducted with live-in care-givers in private households. The evaluation was based on Mayring's content analysis with inductive category formation.

Results and conclusions Permanent presence, communication difficulties and interpersonal conflicts are daily burdens for live-in caregivers. These burdens are associated with impaired mental and physical health in terms of exhaustion, insomnia, increased irritability and depression symptoms. However, livein caregivers also receive support from third parties and distance themselves emotionally and locally from the direct care situation. The feeling of gratitude, the experience of being accepted and also financial security are mentioned as further relevant resources. While the target group can be made visible through resource-intensive recruiting efforts in the research context, the introduction of a common occupational definition is necessary in the political context.

\section{Einleitung}

Aktuell sind in Deutschland nach Schätzungen zwischen 115000 und 300000 sogenannter 24-Stunden-Betreuungskräfte (24h-BK) tätig, die in den Haushalten der von ihnen betreuten Personen arbeiten und wohnen. Mehrheitlich stammen diese Menschen aus mittel- und osteuropäischen Ländern wie Polen, Tschechien, Slowakei, Slowenien, Ungarn, Estland, Lettland, Litauen, Rumänien, Bulgarien oder Kroatien. Sie übernehmen haushaltsbezogene Tätigkeiten wie Reinigung der Wohnung, Zubereitung des Essens aber auch die Betreuung der meist älteren körperlich beeinträchtigten Menschen mit Pflegebedarf [1, 2]. In diesem Arbeitskontext sind 24h-BK besonderen Belastungen ausgesetzt wie Missachtung des Arbeitsund Beschäftigungsrechts [1,3], Nichtzahlung des marktüblichen Mindestlohns [4], nicht vorhandene Qualitätskontrollen [2, 5] und ein unklares Haftungsrisiko im Schadensfall [6]. Lutz (2007) identifiziert als Belastungen am Arbeitsplatz von 24h-BK die „Entgrenzung der Arbeit“, also die zeitliche und räumliche Vermischung von Arbeit und Privatsphäre, aber auch Ängste der Betreuungsperson aufgrund der Illegalität ihrer Tätigkeit und daraus möglicher Auswirkungen auf ihren Aufenthaltsstatus [7]. Karakayali (2010) verweist auf die missbräuchlichen Arbeitssituationen im rechtsfreien Kontext und bestätigt belastende Erfahrungen der Betreuungskräfte durch mangelnde Freizeit und andauernden Zusammenseins mit der pflegebedürftigen Person im selben Haushalt [8].

Zahlreiche Belastungen bestimmen die Tätigkeit von 24h-BK, jedoch berichten sie auch über Ressourcen wie finanzielle Rückführungen (Remittances) an die Familienmitglieder in ihrer Heimat $[9,10]$ oder der Austausch mit anderen Betreuungskräften, die sich in ähnlichen Beschäftigungsverhältnissen befinden [11]. Auch die Wertschätzung der sprachlichen und kulturellen Kompetenz in sprachlich diversen Betreuungsbeziehungen [12] werden von Betreuungskräften als Ressourcen genannt.

Obgleich der Europäische Wirtschafts- und Sozialausschuss verstärkte Forschungsaktivitäten zur Ermittlung der Anzahl, Herkunft, Arbeitsbedingungen und Qualifikationen von 24h-BK fordert, liegen bislang nur wenig wissenschaftliche Erkenntnisse über 24h-BK vor $[2,13]$.

Der Zugang zu 24h-BK, die meist in halblegalen oder irregulären Beschäftigungsverhältnissen mit nicht vorhandenen arbeitsrechtlichen Bestimmungen tätig sind, erweist sich als sehr herausfordernd. Befragungen in deutschen Haushalten fanden in bishe- rigen Studien mit den betreuten Personen und deren Angehörigen statt oder den Dienstleistern oder mit themenbezogenen ExpertInnen aus Politik und Versorgung [1, 5, 13-15].

Das Ziel dieser Untersuchung ist, arbeitsplatzbezogene Belastungen und Ressourcen von ausländischen 24h-BK, die in deutschen Privathaushalten hauswirtschaftliche und personennahe Dienstleistungen für meist ältere und pflegebedürftige Menschen erbringen, zu identifizieren.

\section{Methode}

Die Fragestellungen lauten:

1. „Welche arbeitsplatzbezogenen Belastungen erleben ausländische 24-Stunden-Betreuungskräfte, die in deutschen Privathaushalten arbeiten?"

2. „Welche Ressourcen nutzen sie, um die Belastungen zu bewältigen?"

Es wurde ein qualitativ explorativ deskriptiver Feldforschungsansatz gewählt. Die Berichterstattung der Untersuchung erfolgt in Übereinstimmung mit den „Consolidated criteria for reporting qualitative research“ (COREQ) [16].

\section{Rekrutierung und Setting}

Bei der Auswahl der Stichprobe wurden der nichtdeutsche Geburtsort der Betreuungsperson und 3 Arten von Beschäftigungsverhältnissen berücksichtigt: 1. Direkt (Selbstständigkeit der 24h-BK oder Direktanstellung bei der pflegebedürftigen Person), 2. Indirekt (Arbeitnehmerüberlassung oder -entsendung, 3. Irregulär (undokumentierte Tätigkeit der 24h-BK) [17].

Zur Gewinnung von Betreuungskräften für ein Interview wurden Kontakte zu Vermittlungsagenturen, Onlineportalen, sozialen Netzwerken und Vereinen hergestellt sowie Handzettel an öffentlichen Plätzen verteilt. Potentielle Studienteilnehmerlnnen mussten mindestens 18 Jahre alt und als 24h-BK in Privathaushalten zum Erhebungszeitpunkt tätig sein oder dort gearbeitet haben. Die Interviews wurden in den Haushalten geführt, in denen die Betreuungskräfte tätig sind, aber auch in Cafés, in einer Pizzeria und in den Räumlichkeiten des Instituts unter Gewährleistung der Privatsphäre. Die AutorInnen dieser Studie hatten keine persönliche Beziehung zu den Interviewteilnehmerinnen. 


\section{Datenerhebung}

Die Datenerhebung erfolgte mit semistrukturierten Interviews deren Leitfaden aus den Themen: 1. Ablauf des Arbeitsalltags, 2. Erleben der Arbeit, 3. Belastungen, 4. Ressourcen, 5. Soziodemografische Daten bestand. Der Leitfaden wurde von 3 ExpertInnen für qualitative Sozialforschung und den Autorlnnen dieser Studie geprüft und vor seinem Feldeinsatz einem Pretest unterzogen, indem Interviews mit zwei $24 \mathrm{~h}$-BK geführt wurden; diese sind nicht Bestandteil der vorliegenden Auswertung. Zu Beginn des Interviews wurden die Interviewteilnehmerinnen zum Thema Datenschutz aufgeklärt und entsprechende Einverständniserklärungen zur Studienteilnahme und zur Tonaufnahme schriftlich eingeholt. Der Vertrauensaufbau nahm dabei einen besonderen Stellenwert ein. Die Tonaufnahmen wurden anonymisiert transkribiert. Bei Bedarf wurden die Interviews von qualifizierten SprachmittlerInnen gedolmetscht.

\section{Datenauswertung}

Die Auswertung erfolgte mit MAXQDA $12{ }^{\circledR}$ nach der zusammenfassenden Inhaltsanalyse nach Mayring [18]. Zunächst haben die Autoren und ein weiteres Mitglied der Arbeitsgruppe unabhängig voneinander 2 Transkripte codiert und die Ergebnisse anschließend diskutiert. Das daraus abgeleitete Kategoriensystem wurde bei 2 weiteren Transkripten überprüft und nach entsprechenden finalen Anpassungen auf die verbleibenden Transkripte angewendet.

\section{Ergebnisse}

Insgesamt wurden 58 Unternehmen, die 24h-BK vermitteln, kontaktiert. 30 lehnten die Vermittlung eines Interviewpartners für eine wissenschaftliche Untersuchung ab. Über 3 Anbieter konnten schließlich Kontakte zu 24h-BK hergestellt werden. Darüber hinaus konnten 20 Betreuungskräfte über Anzeigenportale wie auch soziale Netzwerke kontaktiert werden. Schließlich wurden neun Interviews zwischen August und Oktober 2017 geführt. Vier Interviews wurden von qualifizierten Sprachmittlerinnen in der polnischen Sprache gedolmetscht. Die Dauer der Interviews reichte von 62 bis 127 Min. Alle interviewten Personen erhielten eine Aufwandsentschädigung in Höhe von $15 €$ in bar im Anschluss an das Interview. Die Betreuungskräfte stammten aus Polen und Litauen und waren zum Zeitpunkt des Interviews seit einem bis zu fünfzehn Jahre in ihrer Funktion tätig. Einige der 24h-BK haben in ihrem Heimatland studiert oder können ein Abitur nachweisen. Vor Aufnah- me ihrer Tätigkeit als 24h-BK waren sie in ihrer Heimat im Dienstleistungsgewerbe tätig, aber auch als Landwirtin, Unternehmensleiterin oder auch als Hausfrau. Die Beschreibung der Stichprobe ist $\gg$ Tab. 1 zu entnehmen.

\section{Ergebniskategorien}

In > Tab. 2 sind sämtliche Haupt- und Unterkategorien aufgeführt, die induktiv aus dem Datenmaterial abgeleitet wurden.

\section{Belastungen}

\section{Psychische Belastung}

Die Betreuung von KlientInnen mit teilweise schwer ausgeprägten Formen einer Depression oder dementiellen Erkrankung wie auch die Sterbebegleitung, die von Betreuungskräften als Teil ihrer Aufgaben genannt wird, sind belastend. Konflikte in Bezug auf die eigene Verantwortung ergeben sich bei dieser 24h-BK, sobald sie den „richtigen“ Umgang bei bestimmten Krankheitsbildern zu ergründen versucht. „das ist auch Demenz dieser Frau. Sie muss immer laufen und suchen. Und Treppe unten, Treppe oben, bis dritte Stock. Aber von dieser Treppe kann sie runterfallen“ (I 1).

24h-BKs äußern, sich eingesperrt und einsam zu fühlen und erklären dies mit dem Vorherrschen einer „strengen Atmosphäre“ im Betreuungshaushalt (I 3). Der eingeschränkte Kontakt zur Außenwelt, das Umgebensein von älteren und kranken Personen und das Gefühl, ständig verfügbar sein zu müssen, werden in zahlreichen Interviews als belastend genannt.

Eine seit 2 Jahren tätige 24h-BK berichtet: „du kannst nicht sagen: ,ich habe jetzt Feierabend, ich schalte ab'. Du bist immer mit deinem Kopf dabei, immer, Tag und Nacht. Aber ich möchte irgendwann sagen: ,Schluss, ich habe jetzt wirklich Ruhe. Ich brauche nicht reagieren', Aber das geht nicht“ (I 6).

\section{Physische Belastung}

24h-BK berichten von Schlaflosigkeit und erklären, ihr Schlaf hänge vom Rhythmus und dem gesundheitlichen Zustand der Klientlnnen ab. Krankheitsbedingte Anfälle, diagnosebedingte Nachtaktivität der KlientInnen und die regelmäßige Lagerung von bettlägerigen KlientInnen führen dazu, dass $24 \mathrm{~h}-\mathrm{BK}$ auch in den Nächten präsent sein müssen und ruhelose Nächte teils zur Regel gehören.

„Schwere Arbeit mit Patienten mit Demenz. Das ist am schlimmsten Arbeit, weil die eine Nacht gar nicht schlafen. Die Leute schlafen am Tag, nachts sind aktiv“ (I 5).

- Tab. 1 Soziodemografische Daten der Stichprobe.

\begin{tabular}{|c|c|c|c|c|c|c|c|}
\hline $\begin{array}{l}\text { Inter- } \\
\text { view }\end{array}$ & $\begin{array}{l}\text { Alter } \\
\text { (J) }\end{array}$ & $\begin{array}{l}\text { Herkunfts- } \\
\text { land }\end{array}$ & $\begin{array}{l}\text { Höchster } \\
\text { Bildungsabschluss }\end{array}$ & Zuvor ausgeübter Beruf & $\begin{array}{l}\text { als } 24 \mathrm{~h}-\mathrm{Bk} \\
\text { tätig seit (J) }\end{array}$ & $\begin{array}{l}\text { Beschäftigungs- } \\
\text { verhältnis }\end{array}$ & $\begin{array}{l}\text { Kontaktanbahnung } \\
\text { über }\end{array}$ \\
\hline 1 & 46 & Litauen & Studium & Kauffrau & 5 & Irregulär & Online Portal \\
\hline 2 & 49 & Polen & Berufsschule & Hausfrau & 2,5 & Indirekt & Agentur A \\
\hline 3 & 50 & Polen & Studium & Leiterin eines Kleinunternehmens & 1,5 & Direkt & Agentur B \\
\hline 4 & 55 & Polen & Keine & Landwirtin & 12 & Indirekt & Agentur B \\
\hline 5 & 60 & Polen & Studium & Bürohilfe & 15 & Indirekt & Soziales Netzwerk \\
\hline 6 & 38 & Litauen & Abitur & Studentin Psychologie, Köchin & 2 & Indirekt & Online Portal \\
\hline 7 & 55 & Polen & Mittlere Reife & Bürohilfe & 10 & Direkt & Agentur C \\
\hline 8 & 35 & Polen & Abitur & Hotelfachfrau & 1 & Direkt & Agentur C \\
\hline 9 & 57 & Polen & Mittlere Reife & Bürohilfe & 2 & Direkt & Agentur C \\
\hline
\end{tabular}


- Tab. 2 Kategoriensystem der der zusammenfassenden Inhaltsanalyse.

\begin{tabular}{|l|l|}
\hline Belastungen & Ressourcen \\
\hline Psychische Belastung & Selbstwirksamkeit \\
\hline - Art und Schwere der Erkrankung & - Erlernen berufsbezogener Kompetenzen \\
\hline - Einsamkeit & - Treffen klarer Absprachen \\
\hline - Nonstop-Verfügbarkeit & - Räumliche und emotionale Distanzierung \\
\hline Physische Belastung & Gutes Arbeitsklima \\
\hline - Schlaflosigkeit & - Dankbarkeit und Wertschätzung durch Klientln/ Angehörige \\
\hline - Heben und Tragen von Klientlnnen & - Familiäres Verhältnis zu Klientln/ Angehörigen \\
\hline Interpersonelle Konflikte & - Unterstützung durch Pflegedienst, Angehörige, Freunde oder Bekannte \\
\hline - mangelnde sprachliche Verständigung & Motivation \\
\hline - Mangelnde Wertschätzung & - Unterstützung der eigenen Familie durch monetäre Remittances \\
\hline - Teilhabe an familiären Konflikten & - Finanzielle Absicherung der eigenen Person \\
\hline Unklare Rahmenbedingungen & - Menschen helfen können \\
\hline - Mangelnde Privatsphäre & \\
\hline - Falsche und fehlende Informationen über KlientInnnen & \\
\hline - Verpflichtung zur Erledigung nicht vertraglich vereinbarter Aufgaben & \\
\hline
\end{tabular}

Häufig ist die Mobilisierung der KlientInnen mit schwerer körperlicher Anstrengung verbunden: „Wenn die Leute immer im Bett liegen, ist es ganz schwer zu waschen im Bett“ (I 4).

\section{Interpersonelle Konflikte}

Nicht ausreichende Deutschkenntnisse einer 24h-BK können die Verständigung sowohl mit KlientInnen als auch deren Angehörigen zusätzlich erschweren: „Das ist so, wie sich ein Blinder sich mit einem Tauben oder einem Stummen unterhält“ (I 3).

Zudem geben 24h-BK an, nicht akzeptiert oder sogar abgelehnt zu werden und erklären dies damit, dass der/ die die zu betreuende Klientln die eigene Pflegebedürftigkeit nicht akzeptiert oder Angehörige Ratschläge der 24h-BK zum Verhalten gegenüber der Pflegebedürftigen nicht annehmen.

24h-BK erklären, wie Bedienstete behandelt zu werden, die ihre Pflichten im Haushalt zu erfüllen haben. Dies lässt eine funktionierende Beziehungsgestaltung mit den KlientInnen aber auch deren Angehörigen nicht zu und widerspricht dem Wunsch einer 24h-BK, als Familienmitglied anerkannt zu werden. „Wir sind wie Werkarbeiter. Und weil manchmal sagen: ,Du bist Pflegerin. Du musst das machen'. Nein. Ich darf viel machen, aber ich muss nichts“ (I 5).

24h-BK erzählen, dass sie an privaten Angelegenheiten der zu betreuenden Person, der Angehörigen oder der Familie ungewollt teilhaben. „Das sind zwei Schwestern, die haben Zoff wegen Eltern. Da kommt eine, die beschwert sich. Dann kommt andere und ich bin in der Mitte. Ich möchte da nicht stehen. Sie sollen das zwischen sich klären. Sie belasten mich damit“" (I 6).

\section{Unklare Rahmenbedingungen}

24h-BK berichten, kein eigenes Zimmer zu haben und sich so in ihrer Privatsphäre eingeschränkt zu fühlen. Mangelnde Informationen über den Zustand der Person durch die Dienstleisterlnnen und wenig Möglichkeiten zur Absprache hinsichtlich des Tagesablaufs erschweren eine Planung.

Eine 24h-BK führt an, ihre täglich vorgesehenen Pausen seien bei der Tagesplanung nicht berücksichtigt worden und so habe sie wochenlang ohne Pause gearbeitet. Es wird mehrfach von $24 \mathrm{~h}-\mathrm{BK}$ geäußert, Aufgaben übernehmen zu müssen, die vertraglich nicht vereinbart wurden. Dazu gehören die Betreuung weiterer Familienmitglieder, pflegerische Tätigkeiten wie Medikamentengabe, Kochen für ein Familienfest, die psychosoziale Begleitung der Angehörigen oder der Fahrdienst für Familienmitglieder.

\section{Ressourcen}

24h-BK beschreiben zahlreiche Ressourcen, die ihnen helfen, die alltäglichen Belastungen zu bewältigen.

\section{Selbstwirksamkeit}

Eine 24h-BK beschreibt, sich eigeninitiativ über Arzneimittel und Diagnosen zu informieren und auch mit den Ärzten ihres Klienten intensiv über dessen Behandlung zu sprechen, um über dessen Gesundheitszustand informiert zu sein. Das Erlernen und Anwenden von Mobilisierungstechniken zunächst unter Anleitung von Pflegefachkräften erlaubt 24h-BKs, diese Techniken am Klienten zu praktizieren und schließlich selbst körperlich davon zu profitieren. Der aktive Kontakt zu Koordinierungsstellen wird als hilfreich empfunden, denn „wenn die bedürftige Person keine Angehörigen hat, hat die Agentur so eine Koordinierungsstelle, an die man sich wendet und die normalerweise auch ein Problem versucht zu lösen“ (I 8).

Das Treffen klarer Absprachen mit den KlientInnen und deren Angehörigen helfen den Betreuungskräften, den Alltag zu organisieren. „Man muss immer sprechen. Immer fast jede zwei Tage: Was machen wir beide? Was gehört zu Ihrer Aufgabe, was meine Aufgabe?“ (I 1).

Sich aktiv zu distanzieren, hilft den Betreuungskräften, den teilweise sehr belastenden Arbeitsalltag zu bewältigen. Genannt wurden der emotionale Abstand, der sich mit zunehmender Berufserfahrung entwickelt, das bewusste „Etwas nicht persönlich nehmen“, aber auch das bewusste Verlassen der belastenden Situation, indem sich die Pflegekraft in ihr eigenes Zimmer zurückzieht oder eigenen Freizeitaktivitäten nachgeht wie Fahrrad fahren, Lesen oder der Austausch mit Freunden. 


\section{Gutes Arbeitsklima}

Der Ausdruck von Dankbarkeit seitens der Klientlnnen gegenüber 24h-BKs prägt das Verhältnis beider positiv. Als besondere Wertschätzung empfand eine Interviewteilnehmerin die Ausrichtung ihres Geburtstags mit Fest und Torte durch Angehörige ihrer Klientin und die Tatsache, an ihrem Geburtstag nicht arbeiten zu müssen. Als Betreuungskraft von seinen KlientInnen aber auch deren Angehörigen akzeptiert zu werden, wird als wichtige Ressource genannt, wie diese 24h-BK ausdrückt: „die Patientin akzeptiert meine Person. Von Familie akzeptiert werden und gute Kontakte mit Familien “.

Einige Betreuungskräfte erklären, sie hätten ein sehr gutes, teilweise sehr familiäres Verhältnis zu ihren KlientInnen und deren Angehörigen: „Sie fühlte sich wie Mitglied der Familie. Sie hatte Mahlzeiten teilgenommen von der Familie. Die Töchter haben ihr gesagt: ,Setz Dich hin, jetzt unterhältst Du Dich mit uns'. Und das war eine sehr gute Erfahrung“( 7 , gedolmetscht).

Die Unterstützung durch Dritte erleben 24h-BK sehr positiv. Dies helfe ihnen, den Arbeitsalltag bewältigen zu können. Sei es der Pflegedienst, der benutzte Inkontinenzmaterialien oder sonstigen Hausabfall entsorgt oder Bekannte der KlientInnnen, die bei anfallenden Tätigkeiten helfen. Eine $24 \mathrm{~h}$-BK berichtet, sie hätte ihre persönlichen Termine immer wahrnehmen können, weil die Tochter der Klientin dann die Betreuung übernommen habe.

\section{Motivation}

Schicksale in der Heimat, wie der Verlust des Arbeitsplatzes oder Frühverrentung und dadurch entstehende finanzielle und soziale Sorgen, werden als Anlässe genannt, in Deutschland der Tätigkeit der 24- Stunden-Betreuung nachzugehen. Diese 24h-BK erklärt: "Ihr Ehemann ist gestorben und sie wollte ihrer Tochter finanziell helfen" (I 9, gedolmetscht).

Ein umfassender Krankenversicherungsschutz, eine legale Beschäftigung und ein geregeltes Einkommen werden von 24h-BK als positiv erlebt. Teilweise seien sie in ihrer Heimat in diesen Bereichen schlechter gestellt und daher empfänden sie ihre Beschäftigung in Deutschland wie auch einen dort wesentlich höheren Verdienst als Absicherung.

Den Wunsch, sich in helfenden Berufen zu engagieren und so seine Bestimmung zu finden, drückt diese 24h-BK aus: „Ja, das für mich ist gute Arbeit. Alles mit alter Frau oder altem Mann. Hilfe, ich liebe Hilfe“ (I 2). Ihren Wunsch, im Heimatland als Krankenschwester zu arbeiten, nicht erfüllen zu können, motivierte eine $24 \mathrm{~h}-\mathrm{BK}$, in Deutschland als Betreuungskraft zu arbeiten - denn so könne sie trotzdem anderen Menschen helfen.

\section{Diskussion}

Die vorliegende Studie identifiziert arbeitsplatzbezogene Belastungen und Ressourcen von ausländischen 24h-BK in deutschen Privathaushalten. Aufgrund in Deutschland kaum vorhandener Studien wurde in dieser Studie ein qualitativer Forschungsansatz verfolgt und dabei 24h-BK direkt befragt. Die Nonstop-Verfügbarkeit, Schlaflosigkeit, Verständigungsschwierigkeiten und interpersonelle Konflikte sind alltäglich gegenwärtig und werden in Untersuchungen von Karakayali $(2010)$ bestätigt $[8,19]$. 24h-BK äußern, sie seien physisch und psychisch erschöpft und erhöht reizbar, so dass sie sich z. T. selbst nicht mehr wahrnehmen können. Dennoch empfinden sie die Tätigkeit als 24h-BK als einzigartige Chance, sich zu verwirklichen, indem sie bedürftigen Menschen helfen und dabei auch Geld verdienen können; entsprechend relativieren sie die wahrgenommen teils extremen psychischen und physischen Belastungen. Timonen und Doyle (2010) berichten über irische Betreuungskräfte, die ihre Arbeitsverhältnisse als teilweise ausbeuterisch empfinden und diese aber aufgrund einer moralischen Verpflichtung zur Unterstützung ihrer KlientInnen als gegeben akzeptieren [20]. Die körperlich anstrengende Arbeit und der täglich intensive Umgang und Kontakt mit teilweise schwer psychisch erkrankten Menschen erzeugt bei 24h-BK nach eigener Aussage starke Rückenbeschwerden und Depressionen. Kaufmann et al. (1982) bestätigen aus arbeitspsychologischer Sicht den Zusammenhang von Arbeitsbelastungen und Unzufriedenheit, erhöhter Reizbarkeit sowie Erschöpfung [21].

Eine räumliche und emotionale Distanzierung hilft 24h-BK, sich von einer belastenden Betreuungssituation abzugrenzen. Das $\mathrm{Er}$ leben von Dankbarkeit, das Gefühl von seinen KlientInnen und gegebenenfalls deren Angehörigen akzeptiert zu werden, erleben 24h-BK als wertschätzend. Die Möglichkeit, eigene Freiheiten zu haben und einen Raum, um sich zurückziehen zu können, tragen entscheidend zur Arbeitszufriedenheit von 24h-BK bei. Kniejska (2016) hat in ihren Interviews mit 24h-BK gezeigt, dass diese dann eine hohe Arbeitszufriedenheit aufweisen, wenn sich ihre Tätigkeit positiv auf den physischen und psychischen Zustand der Klientlnnen auswirkt, bspw. wenn durch permanente Mobilisierung bei anfänglicher Bettlägerigkeit der Klient oder die Klientin wieder Treppensteigen kann [15].

Die in Deutschland geführte Diskussion über prekäre Arbeitsverhältnisse auch im Kontext einer zu geringen Entlohnung $[2,7,13-15]$ wird in der vorliegenden Studie durch die interviewten 24h-BK nicht bestätigt. Ganz im Gegenteil berichten sie, ihr Gehalt sei um ein Vielfaches höher als das in ihrem Heimatland und dies sei einer der Gründe für die Aufnahme einer Tätigkeit in Deutschland.

\section{Schwächen und Stärken der Studie}

Die Rekrutierung von TeilnehmerInnen in qualitativen Studien ist anfällig für Selektionseffekte. In dieser Studie erfolgte die Auswahl der TeilnehmerInnen über mehrere Institutionen. Dabei ist die Kontaktanbahnung über solche Vermittlungsagenturen zu nennen, bei denen zunächst mehrere Instanzen befragt werden mussten, bevor ein Kontakt zu einer 24h-BK hergestellt werden konnte. Die Transkripte der Interviews wurden den 24h-BK weder zur Korrektur oder möglichen Kommentierung vorgelegt noch wurden letztgenannte in den Prozess der Manuskripterstellung miteinbezogen.

Die klaren Stärken dieser Erhebung zeigen sich durch den intensiven Diskurs zwischen den StudienautorInnen bei der Kategorienbildung. Der Einsatz von Dolmetscherinnen ermöglichte den interviewten 24h-BK in ihrer Muttersprache zu kommunizieren. Die Rückmeldung einiger 24h-BK, das Interview als Wertschätzung für ihre Tätigkeit empfunden zu haben, bestätigt den Sinn des sehr hohen Rekrutierungsaufwands.

24h-BK sind im Forschungssinne schwer erreichbare Personengruppen. Der notwendige hohe zeit- und ressourcenintensive Aufwand ist zur Herstellung einer Interviewsituation bei entsprechenden Forschungsvorhaben zu berücksichtigen. 
Eine Diskussion über die prekären Beschäftigungsverhältnisse von 24h-BK kann nur dann im Licht des ratifizierten Übereinkommens Nr. 189 der ILO auf politischer und gesellschaftlicher Ebene erfolgen, wenn diese Gruppe von Menschen auch definierbar und damit wissenschaftlich und politisch erreichbar ist. Die Einführung einer gemeinsamen Berufsdefinition ist daher notwendig [22] und wird auch in anderen Studien in Europa bereits gefordert [23].

\section{Interessenkonflikt}

Die Autoren geben an, dass kein Interessenkonflikt besteht.

\section{Literatur}

[1] Neuhaus A, Isfort M, Weidner F. Situation und Bedarfe von Familien mit mittel- und osteuropäischen Haushaltshilfen.Deutsches Institut für angewandte Pflegeforschung e.V.; Köln: 2009. Im Internet: https:// www.dip.de/fileadmin/data/pdf/material/Endbericht_Haushaltshilfen. pdf; Stand: 31.05.2018

[2] Böning M, Steffen M. Migrantinnen aus Osteuropa in Privathaushalten: Problemstellungen und politische Herausforderungen. ver.di-Bundesverwaltung (März 2014). Im Internet: https://gesundheit-sozialeshessen.verdi.de/ + + file + +5374a0796f6844065400076a/download/ Migrantinnen \%20in \%20Privathaushalten \%20FINAL.pdf; Stand: 16.12.2018

[3] International Labour Organization (ILO). Domestic Workers (2018). Im Internet: http://www.ilo.org/global/topics/care-economy/domesticworkers/lang--en/index.htm; Stand: 01.06.2018

[4] Martin-Matthews A. Employers, home support workers and elderly clients: identifying key issues in delivery and receipt of home support. Healthcare Quarterly 2008; 11: 69-76

[5] Prochazkova L, Rupp B, Schmid T. Evaluierung der 24-Stunden-Betreuung. Endbericht. SFS - Sozialökonomische Forschungsstelle, Wien (Juli 2008). Im Internet: https://www.sozialministerium.at/cms/site/ attachments/8/9/2/CH3434/CMS1458570732748/evaluierung_ der_24-stunden-betreuung_(endbericht).pdf; Stand: 31.05 .2018

[6] von Kondratowitz $\mathrm{H}$-J. Die Beschäftigung von Migranten/innen in der Pflege. Zeitschrift für Gerontologie und Geriatrie 2005; 38: 417-423

[7] Lutz H. Vom Weltmarkt in den Privathaushalt. Die neuen Dienstmädchen im Zeitalter der Globalisierung. Unter Mitarbeit von Susanne Schwalgin. 2. überarbeitete Auflage. Leverkusen: Verlag Barbara Budrich 2008; 151-170

[8] Karakayali J. Die Regeln des Irregulären - Häusliche Pflege in Zeiten der Globalisierung. In Scheiwe K, Krawietz J, Hrsg. Transnationale Sorgearbeit. Rechtliche Rahmenbedingungen und gesellschaftliche Praxis. Wiesbaden: VS Verlag für Sozialwissenschaften; 2010: 151-170

[9] Humphries N, Brugha R, McGee H. Sending money home: a mixedmethods study of remittances by migrant nurses in Ireland. Human Resources for Health doi:10.1186/1478-4491-7-66
[10] Larsen JA, Allan HT, Bryan K et al. Overseas Nurses 'Movitations for Working in the UK: globalization and life politics. Work, Employment and Society 2005; 19: 349-368

[11] Covell C, Neiterman E, Bourgeault IL. Scoping review about the professional integration of internationally educated health professionals. Human Resources for Health doi:10.1186/s12960-016-0135-6

[12] Omeri A, Atkins K. Lived experiences of immigrant nurses in New South Wales, Australia: searching for meaning. International Journal of Nursing Studies 2002; 39: 495-506

[13] Hielscher V, Kirchen-Peters S, Nock L. Pflege in den eigenen vier Wänden: Zeitaufwand und Kosten. Pflegebedürftige und ihre Angehörigen geben Auskunft. edition Hans-Böckler-Stiftung. Study Nr. 363. (Juni 2017). Im Internet: https://www.boeckler.de/pdf/p_study_hbs_363.pdf; Stand: 01.06.2018

[14] Emunds B, Schacher U. Ausländische Pflegekräfte in Privathaushalten. Abschlussbericht zum Forschungsprojekt Frankfurter Arbeitspapiere zur gesellschaftsethischen und sozialwissenschaftlichen Forschung (November 2012). Im Internet: https://nbi.sankt-georgen.de/assets/ typo3/redakteure/Dokumente/FAgsFs/Pflege_HBS_Endfassung.pdf; Stand: 01.06.2018

[15] Kniejska P. Migrant Care Workers aus Polen in der häuslichen Pflege. Zwischen familiärer Nähe und beruflicher Distanz. Wiesbaden: VS Verlag für Sozialwissenschaften; 2016

[16] Tong A, Sainsbury P, Craig J. Consolidated criteria for reporting qualitative research (COREQ): a 32-item checklist for interviews and focus groups. International Journal for Quality in Health Care 2007; 19: 349-357

[17] Knopp L. Gesetzlicher Mindestlohn auch für osteuropäische Pflegehilfen - legale Modelle versus Schwarzmarkt. Neue Zeitschrift für Arbeitsrecht 2015; 14: 851-853

[18] Mayring P. Einführung in die qualitative Sozialforschung 6. überarbeitete Ausgabe. Weinheim. Beltz; Verlag: 2016

[19] Mehta KK, Leng TL. Experiences of Formal and Informal Caregivers of Older Persons in Singapore. Journal of Cross-Cultural Gerontology 2017; 32: 373-385

[20] Timonen V, Doyle M. Migrant Care Workers' Relationships with Care Recipients, Colleagues and Employers. European Journal of Women's Studies 2010; 17: 25-41

[21] Kaufmann I, Pornschlegel H, Ivars U. Belastungen und Stress bei der Arbeit. Körperliche und psychische Beanspruchung, Gesundheit, Erholungspausen. In Zimmermann L, Hrsg. Humane Arbeit - Leitfaden für Arbeitnehmer, Band 5. Reinbek bei Hamburg: Rowohlt; 1982

[22] European Economic and Social Committee (EESC). The rights of live-in care workers. Opinion. (21.09.2016). Im Internet; https://webapi.eesc. europa.eu/documentsanonymous/eesc-2016-00941-00-00-ac-tra-en. docx; Stand: 01.07.2018

[23] Castagnone E, Salis E, Premazzi V. Promoting integration for migrant domestic workers in Italy. International and European Forum of Research on Immigration (FIERI).International Labour Office (2013). Im Internet: http://www.ilo.org/wcmsp5/groups/public/---ed_protect/--protrav/---migrant/documents/publication/wcms_222290.pdf; Stand: 02.06.2018 